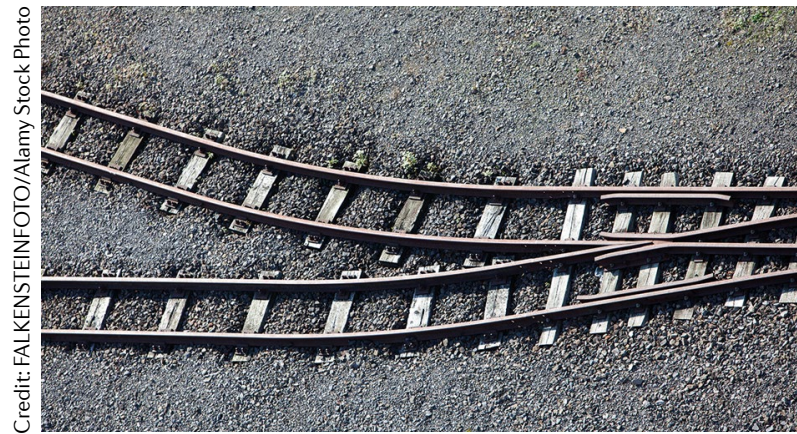

ADDICTION

\section{Pathways to contextual control}

Context has an important role in addictive disorders: exposure to contexts previously associated with drug use promotes relapse, whereas exposure to contexts associated with abstinence can protect against such relapse. In a new study, Gibson et al. describe the opposing roles of two nucleus accumbens output pathways in context-specific relapse of alcohol seeking in rats.

The shell of the nucleus accumbens (AcbSh) contributes to the contextual control of both relapse and abstinence, but how it mediates these opposing effects is unknown. Here, Gibson et al. found that two discrete populations of dopamine 1 receptorexpressing neurons in AcbSh project to the ventral tegmental area (VTA) and lateral hypothalamus (LH), where they make monosynaptic inhibitory connections with GABAergic neurons.

To investigate the contributions of these projections to the promotion or prevention of relapse, the authors used a well-established model of context-induced reinstatement. Rats were trained to respond with a particular behaviour to receive an alcoholic reward in one context, and then extinction training took place in a second context, in which the response produced no reward. As expected, animals re-exposed to the initial context rapidly reinstated their behavioural response; however, optogenetic inhibition of VTA-projecting AcbSh neurons or chemogenetic excitation of the VTA GABAergic neurons to which they project prevented this reinstatement, indicating that the AcbSh-VTA projection is necessary for context-induced relapse.

Optogenetic inhibition of the AcbSh-LH pathway had no effect on context-induced reinstatement; however, optogenetic excitation of this pathway prevented reinstatement. Furthermore, inhibition of the AcbSh-LH pathway or chemogenetic excitation of LH GABAergic neurons prevented the extinction of drug-seeking behaviour in a single-context paradigm, indicating a role for this pathway in the prevention of relapse.

This study demonstrates that distinct output pathways of a single brain subregion mediate the opposing effects of context on drug-seeking behaviour. Understanding these complex control pathways may provide new avenues for their therapeutic manipulation in addictive disorders.

Katherine Whalley

ORIGINAL ARTICLE Gibson, G. D. et al. Distinct accumbens shell output pathways promote versus prevent relapse to alcohol seeking. Neuron https://doi.org/10.1016/j. neuron.2018.03.033 (2018)

GLIA

\title{
Synaptic nibbling
}

Microglia are proposed to remodel neuronal synapses by pruning them via phagocytosis. However, Weinhard et al. now report that, rather than removing whole synapses, microglia prune presynaptic structures through a selective partial phagocytic process termed trogocytosis, or 'nibbling'.

The authors analysed fixed hippocampal tissue from postnatal day 15 (P15) mice using quantitative confocal microscopy as well as correlative light and electron microscopy, and found no evidence of phagocytosis of dendritic spines by microglia. Instead, microglia only pruned presynaptic structures - of $250 \mathrm{~nm}$ on average - by trogocytosis. Interestingly, trogocytosis seemed to involve the 'sinking' of presynaptic structures into the microglial cytoplasm prior to membrane closure, whereas pseudopodia, a hallmark of phagocytosis, were not observed.

Next, the researchers used light-sheet fluorescence microscopy of hippocampal slice cultures (derived from P4 mice and maintained for up to 3 weeks) to study the dynamics of microglia-synapse interactions. These cultures recapitulated in vivo microglial physiology, as the dynamics of extension and retraction of microglial processes were consistent with those in previous in vivo studies. Importantly, the authors detected only partial engulfment of presynaptic (and not postsynaptic) structures; these trogocytic events were rapid, often completing in $<3 \mathrm{~min}$. Interestingly, unlike the phagocytic clearance of apoptotic cells by microglia in the developing hippocampus, trogocytosis of synaptic structures by microglia does not involve the complement system, as the authors found no defects in

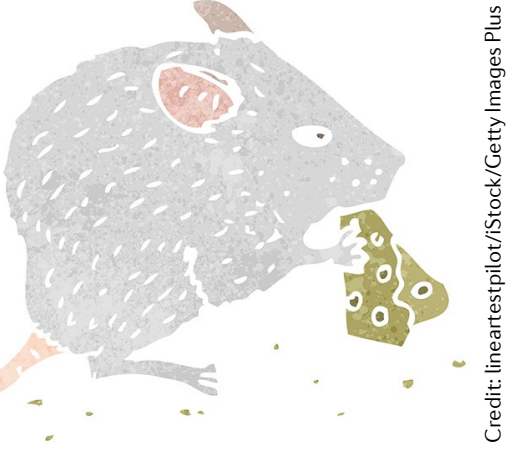

trogocytosis in mice deficient in the CR3 complement receptor.

Further analysis of the light-sheet fluorescence microscopy data revealed that microglia contacted dendritic spines often and briefly, and multiple contacts with the same spine were observed. Although 10\% of spines contacted by microglia disappeared during imaging, these spines were not in contact with microglia when they disappeared. Moreover, there was no difference in the frequency of disappearance of microglia-contacted versus non-contacted spines. Together, these data suggest that microglia do not induce spine elimination in this preparation.

Intriguingly, transient contacts between spines and microglial processes often resulted in formation of filopodia from the spine head, which extended towards the microglial process, whereas no spine-head filopodia formed from non-contacted spines. Additionally, contact with the microglial process sometimes resulted in dramatic stretching of spines, suggesting that microglia could influence the morphology of spines.

These data suggest that, in the mouse postnatal hippocampus, microglia seem to have a more nuanced role in synaptic remodelling, including the trogocytosis of axonal structures and the restructuring of postsynaptic sites.

Grant Otto

ORIGINAL ARTICLE Weinhard, L. et al. Microgli remodel synapses by presynaptic trogocytosis and spine head filopodia induction. Nat. Commun. 9 , 1228 (2018) 